\title{
Voltage-gated Sodium Channels and Blockers: An Overview and Where Will They Go?*
}

\author{
Zhi-mei LI ${ }^{1}$, Li-xia CHEN#, Hua LI ${ }^{1 \#}$ \\ ${ }^{1}$ Hubei Key Laboratory of Natural Medicinal Chemistry and Resource Evaluation, School of Pharmacy, Tongji Medical College, \\ Huazhong University of Science and Technology, Wuhan 430030, China \\ ${ }^{2}$ Wuy a College of Innovation, Key Laboratory of Structure-Based Drug Design \& Discovery, Ministry of Education, Shenyang \\ Pharmaceutical University, Shenyang 110016, China
}

(C) The Author(s) 2020, corrected publication October 2020

\begin{abstract}
Summary: Voltage-gated sodium (Nav) channels are critical players in the generation and propagation of action potentials by triggering membrane depolarization. Mutations in Nav channels are associated with a variety of channelopathies, which makes them relevant targets for pharmaceutical intervention. So far, the cryoelectron microscopic structure of the human Nav1.2, Nav1.4, and Nav1.7 has been reported, which sheds light on the molecular basis of functional mechanism of Nav channels and provides a path toward structure-based drug discovery. In this review, we focus on the recent advances in the structure, molecular mechanism and modulation of Nav channels, and state updated sodium channel blockers for the treatment of pathophysiology disorders and briefly discuss where the blockers may be developed in the future.
\end{abstract}

Key words: voltage-gated sodium channels; blockers; Nav channel structures; channelopathies

Life did not come into existence until living organisms were enclosed by one or more membranes which cut them off from the chaotic world at a molecular level. Whereas, to respond to various cellular housekeeping reactions or stimuli given to the cell in the environment, the living cells developed the ability to overcome this barrier ingeniously by providing pathways to link the extracellular medium, cytoplasm and the contents of intracellular organelles. Indeed, transporting proteins embedded in membranes including pumps, channels and transporters are vitally important parts in these "pathways" to fulfill substances traveling across the membrane for the processes of energy transduction and cell excitability. Being the crucial components of living cells, ion channels regulate the passage of ions across the membranes and perform the primal functions to form the basis for the elaborate development of electrical properties that allow perception, thought, and motion. That is to say, studded with these ion channels in the membranes, the

The original version of this article was revised due to a retrospective Open Access order.

Zhi-mei LI, E-mail: li zhimei@hust.edu.cn

\#Corresponding authors, Li-xia CHEN,E-mail:syzyclx@163. com; Hua LI, E-mail: li_hua@hust.edu.cn

${ }^{*}$ This work was supported by the National Natural Science Foundation of China (Nos. 81473254, 81773637, 81773594, U1703111), and the Fundamental Research Fund for the Central Universities (No. 2017KFYXJJ151). excitable cells could transduce electrical and chemical stimuli into currents of charged species.

In this review, we focus on voltage-gated sodium (Nav) channels, which selectively conduct sodium ions movement in response to variations of membrane potentials ${ }^{[1]}$. Defective Nav channels can give rise to channel malfunctions, leading to both neuronal hypo- and hyperexcitability, accordingly associated with acquired or inherited disorders (sodium channelopathies) ${ }^{[2,3]}$. To date, more than 1000 point mutations of Nav channels have been detected in the central nervous system (CNS), the peripheral nervous systems (PNS), heart, skeletal muscles and cancer cells, which expanded our knowledge of the role of Nav channels in the wide spectrum of diseases such as epilepsy, migraine, pain, myotonic syndromes, diabetes, autism, cardiovascular diseases and so on ${ }^{[3-7]}$. For example, Nav1.7 subtype, preferentially expressed in the olfactory epithelium, sympathetic neurons, and dorsal root ganglion sensory neurons, plays a key role in pain transmission ${ }^{[8]}$. Dysfunction of Nav1.7 may predispose people to lots of disordered diseases like indifference to pain ${ }^{[9]}$. Therefore, Nav channels display major targets for various clinical therapeutics and natural toxins ${ }^{[10,11]}$. To date, X-ray and cryoelectron microscopic (cryo-EM) structures of Nav channels have been gradually reported. In 2011, the first X-ray protein structure of sodium channel from Arcobacter butzleri (NaVAb) was obtained ${ }^{[12,13]}$. In 2012, NaVRh, a 
sodium channel from Alpha proteobacterium HIMB114 (Rickettsiales sp. HIMB114) ${ }^{[14]}$ and NaVMs, a sodium channel from Magnetococcus sp. (strain MC-1) ${ }^{[15]}$ were reported. The eukaryotic Nav channels were structurally unrevealed until 2017. The resolutions of cryo-EM structures of a Nav channel from American cockroach (NavPaS) and a prototypal Nav channel from electric eel (EeNav1.4) in complex with the $\beta 1$ subunit were determined to be 3.8 and $4.0 \AA$, respectively, displaying distinct conformations ${ }^{[16,17]}$. In 2018, the insect NavPaS in complex with a gating modifier toxin Dcla was identified ${ }^{[18]}$, and scientists reported the cryo-EM structure of human Nav1.4- $\beta 1$ complex at $3.2 \AA^{[19]}$. In 2019 , cryo-EM structures of human Nav1.7 in complex with auxiliary subunits and animal toxins ${ }^{[20]}$, and human Nav1.2- $\beta 2-\mu$-conotoxin KIIIA complex ${ }^{[21]}$ were reported. These structures could help gain insight into the molecular basis of functional mechanism of Naw channels and provide a path toward structure-based drug discovery.

\section{MOLECULAR STRUCTURE OF NAV CHANNELS}

Nav channels are formed by a central $\alpha$-subunit and one or more auxiliary $\beta$-subunits. As shown in fig. 1 , the $\alpha$-subunit constitutes two functionally entities, the ion-conducting pore domain and the voltage-sensing domains (VSDs) ${ }^{[5,22]}$. $\alpha$-subunit contains four repeated units of homology ( I -IV or D1-D4), each developed by $\alpha$-helical transmembrane segments $(\mathrm{S} 1-\mathrm{S} 6)^{[23]}$. In mammals, nine functional $\alpha$-subunits (Nav1.1-Nav1.9) have been cloned $^{[24]}$. All $\alpha$-subunits share nearly

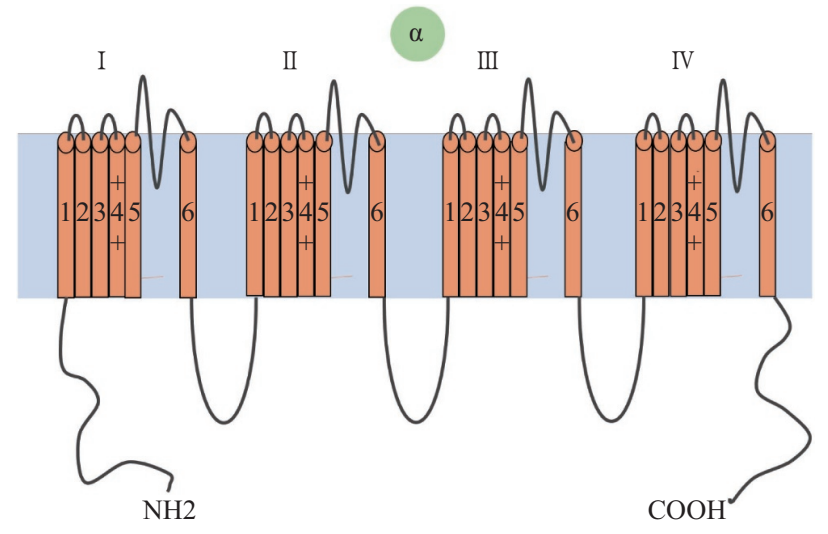

Fig. 1 Voltage-gated sodium (Nav) channel $\alpha$-subunit topology The $\alpha$-subunit contains four homologous domains ( I-IV) connected by intracellular linkers, each developed by $\alpha$-helical transmembrane segments (S1-S6). Voltagesensing domain (VSD) is constituted within S1-S4, which control the gating. The fourth hydrophobic segment, S4, in each domain contains positively charged amino acids (arginine or lysine) and functions as voltage sensors. Segments S5, S6, and the connecting pore-loops form the channel pore. The intracellular loop between domain III and domain IV contains the IFM (isoleucine, phenylalanine, and methionine) domain required for channel inactivation. homologous structure topology, which makes it hard to design isoform-selective blockers for particular Nav channel ${ }^{[25]}$. The expression of Nav channel $\alpha$-subunits is both cell and tissue specific. Nav1.1, Nav1.2, and Nav1.3 subunits are present in the CNS. Nav1.4 is the main subunit expressed in the skeletal muscle. Nav1.5 subunits are expressed primarily in cardiac muscle. Nav1.6 is located in both CNS and PNS. Proteins Nav1.7-Nav1.9 are mostly restricted to PNS. According to the sensitivity to the tetrodotoxin (TTX) isolated from the puffer fish, the nine $\alpha$-subunit can be classified into two groups. Namely, Nav1.1, Nav1.2, Nav1.3, Nav1.4, Nav1.6, and Nav1.7 are TTX-sensitive as they are blocked by nanomolar concentrations of TTX. Nevertheless, Nav1.5, Nav1.8 and Nav1.9 are TTX-resistant channels as they are inhibited by much higher TTX concentrations.

There are three functional states for Nav channels: a closed resting state, an activated state, and a fast inactivation state. Specifically, the structure basis for voltage sensitivity is constituted within S1-S4, which controls the gating, while S5-S6 segments enclose the pore domain and the sequences between them assemble the extracellular domains, the selectivity filter (SF), and the SF-supporting helices $\mathrm{P} 1$ and $\mathrm{P} 2^{[23,26,27]}$. Compared to calcium and potassium channels, Nav channels possess a highly asymmetric SF. Four amino acid residues including aspartate, glutamate, lysine, and alanine (DEKA) which are located in repeats I, II, $\mathrm{III}$, and IV, respectively in the $\mathrm{P}$ region are crucial for sodium selectivity ${ }^{[28]}$. In particular, the fourth hydrophobic segment, S4, one in each domain of Nav channels heterotetramer, has a well-conserved motif of positively charged amino acids (arginine or lysine), which keeps the highly charged secondary structure poised to respond to the voltage changes by moving along the membranes in a stepwise manner. The mode of helical screw motion of S4 contributes to the dynamic process of the gate in the S6 segment ${ }^{[29,30]}$. Movements of the charged residues are coupled to the opening of the pore domain upon membrane depolarization, and subsequently sodium ions travel across cell membrane. Mutagenesis studies carried out for Nav channels indicated that the shape and chemical complementarity between S4-S5 linker and the S6 C-term could work for the gating properties. Inactivation is an important functional feature of Nav channels for preventing a runaway of potential stability. There is evidence indicating that the conserved intracellular linker between domain III and domain IV is critical for fast inactivation $^{[16]}$. A set of hydrophobic amino acids (isoleucine, phenylalanine, methionine and threonine) called IFMT motif is involved in rapid inactivation by closing the entrance of the pore ${ }^{[3]}$. Many details on the binding site for the inactivation are still controversial. Nonetheless, it is commonly accepted that the Nav 
channels inactivation comes from its specific pore sequences. With evolution and structural studies carried out, this distinctiveness may endow it with the exclusive privilege as an excellent drug target.

$\beta$-subunits are transmembrane proteins, and each one is composed of a single $\alpha$-helix stretching across the membrane. With a large extracellular $\mathrm{N}$ terminus, Nav channels $\beta$-subunit displays an immunoglobulin outer segment to bind external proteins and modulates membrane trafficking, channel gating kinetics, and voltage dependence.

In 2017, the first near-atomic resolution structure of eukaryotic Nav channel, NavPaS, reveals that its intracellular gate is firmly closed and the side walls are sealed without fenestrations, reminiscent of what may not represent an inactivated state ${ }^{[16]}$ (fig. 2). Nevertheless, the VSDs show "up" conformations but are distinct from entirely activated conformations. Meanwhile, the intact III-IV linker is sequestered by the globular C-terminal domain (CTD), which is unlikely to be able to conduct its inactivating function. In addition, the IFM motif that is crucial for fast inactivation is replaced by Ala/Thr/Asp in NavPaS. Nonetheless, the near-atomic resolution structure of a single chain eukaryotic Nav channel was established as an important foundation for investigating the function-disease mechanisms of Nav channels and for structure-guided drug development. In other words, the structure opens a new chapter for mechanistic investigation of Nav channels and drug discovery for Nav channelopathies. In the same year, the structure of EeNav1.4 was reported (fig. 3). In contrast with NavPaS, EeNav1.4 exhibits features as follows. (i) The intracellular gate is kept open by a digitoninlike molecule. (ii) Whereas side groups could not be assigned for VSDs in repeats I and II, the resolved VSDIII and VSDIV both exhibit "up" conformations. (iii) The IFM motif is positioned between the inner S6 segments and the outer S4-S5 linkers in repeats III and $\mathrm{IV}^{[19]}$. It should be noted that electrophysiological states of the two sodium channels are yet to be defined for their structures in the absence of other reference structures.

Almost all venoms from nature contain toxins that regulate the activity of Nav channels in order to paralyze prey or predators. Neurotoxins like TTX and saxitoxin (STX) function as pore blockers, modulate the channel in a particular stage of the gating cycle through interacting with one or more $\mathrm{VSDs}^{[32]}$. Scientists presented structures of the insect Nav channel NavPaS bound to a gating modifier toxin Dc1a at 2.8 angstromresolution and in the presence of TTX or STX at 2.6$\AA$ and 3.2- $\AA$ resolution, respectively, which elucidated the molecular basis for the insect selectivity of Dcla and the subtype-specific binding of TTX or STX to Nav channels ${ }^{[18]}$ (fig. 4). In the structure of NavPaS-

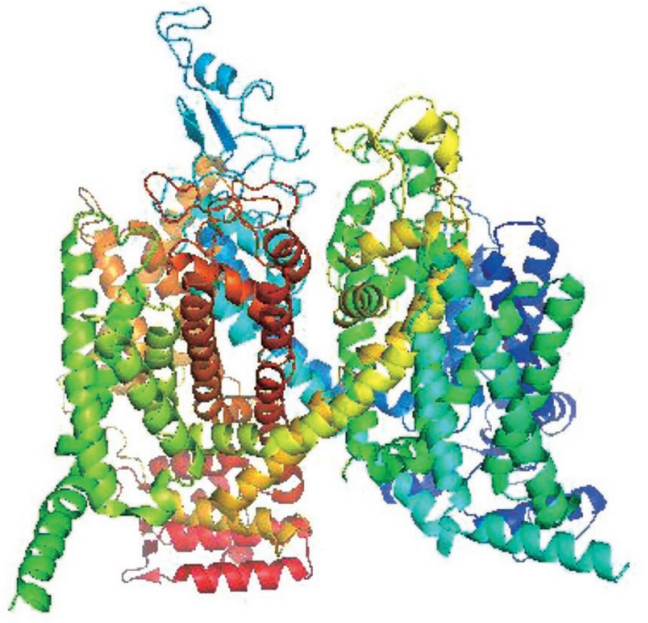

Fig. 2 The overall structure of NavPaS, a eukaryotic voltagegated sodium channel from American cockroach

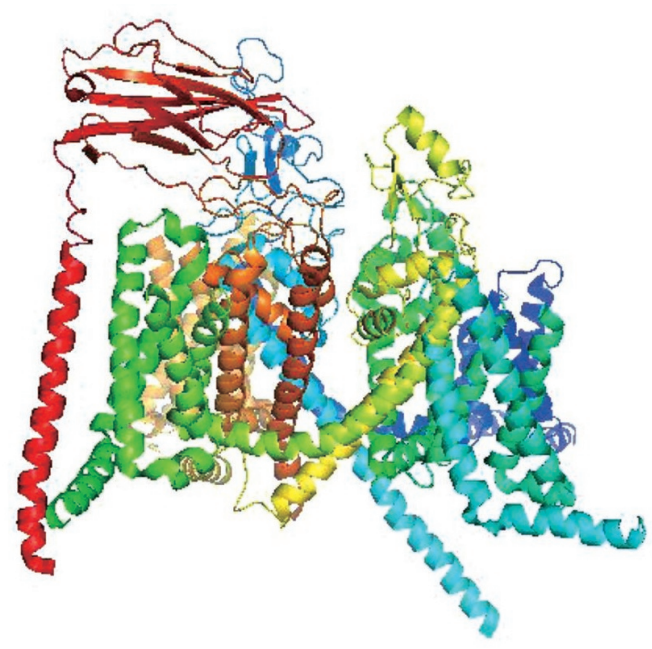

Fig. 3 The overall structure of EeNav1.4, the Nav channel from electric eel, in complex with the $\beta 1$ subunit at $4.0 \mathrm{~A}^{\circ}$ resolution

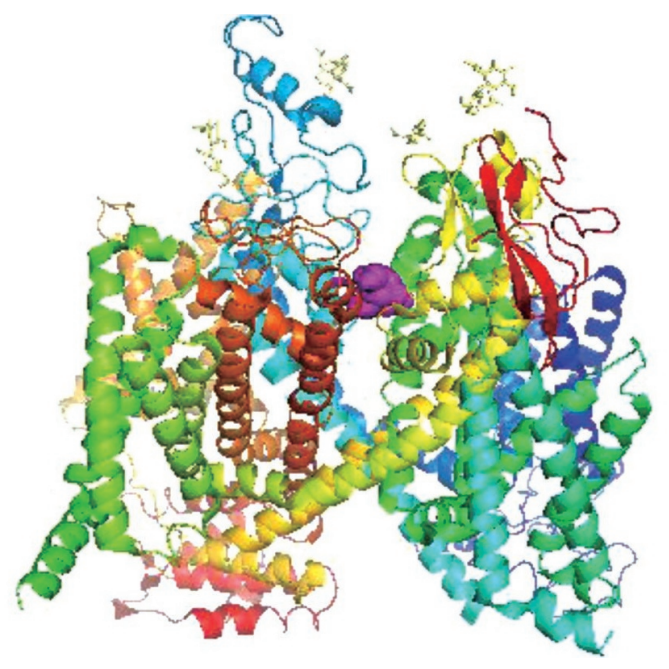

Fig. 4 The Overall structure of the NavPaS-Dc1a-TTX complex The sugar moieties are shown as yellow sticks. TTX, shown as purple balls. Because the three overall structures (NavPaS-Dc1a-TTX, NavPaS-Dc1a-STX, NavPaS-Dc1a) are nearly identical, only one is shown as a representative. 
Dc1a complex, VSD II showed an important role in binding the gating modifier toxin (GMT). In addition, it displays the interaction between the toxin, the S5 III pore-domain and the extracellular dome above the pore.

To further study the structure-function relationship of Nav channel, scientists reported the cryo-EM structure of human Nav1.4- $\beta 1$ complex at $3.2 \AA^{[19]}$ (fig. 5 ). This accurate structure provides important insight into the molecular basis for series of process of sodium function including $\mathrm{Na}+$ permeation, electromechanical coupling, asynchronous activation, and fast inactivation of the four repeats, which provided an accurate template to map mutations linked with diseases such as myotonia and periodic paralysis. In this structure, Voltage sensing involves 4-6 Arg/Lys residues on S4 of the VSD. This helix moves "up" due to the changes of the membrane potential, which opens the channel in the end. All four VSDs exhibit up conformations. IFM motif between repeats III and IV inserts into the hydrophobic cavity which is enclosed by S4-S5 segments and the S6 in repeats III and IV. This allosteric blocking mechanism for fast inactivation is verified by the interactions between functional residues and disease mutations. The structure possesses remarkable physiological and pathophysiological significance. Meanwhile, it bridges molecule basis of functional mechanism of voltagegated sodium channels with future structure-based drug discovery for the treatment of channelopathies.

Studies revealed that the four $\beta$ subunits ( $\beta 1$ to $\beta 4$ ) affect the properties of Nav1.7 channel. $\beta 1$ and $\beta 2$ are commonly co-expressed with the $\alpha$ subunit of Nav1.7 for biophysical characterization ${ }^{[33]}$. Recently, the cryo-EM structures of the human Nav1.7- $\beta 1-\beta 2$ complex bound to two combinations of gating modifier toxins (GMTs) and pore blockers, saxitoxin with huwentoxin-IV (HWTX-IV) and tetrodotoxin (ProTx- II ) with protoxin- II , both determined at 3.2

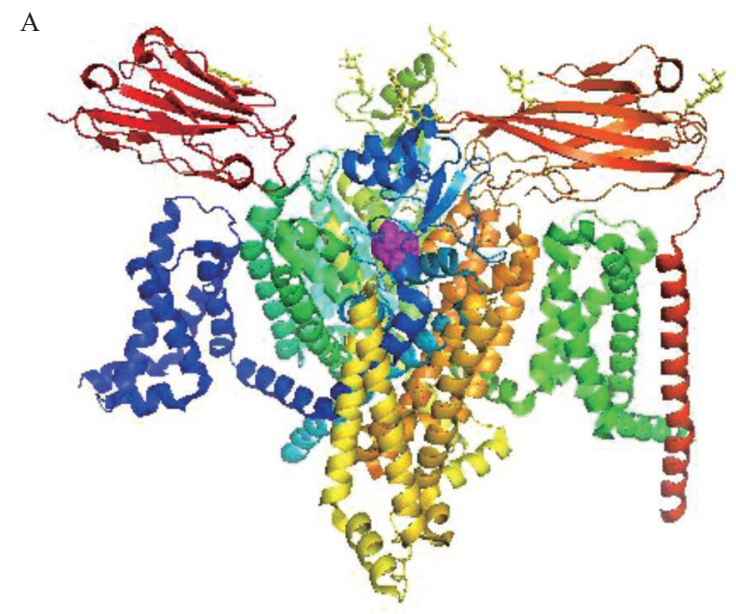

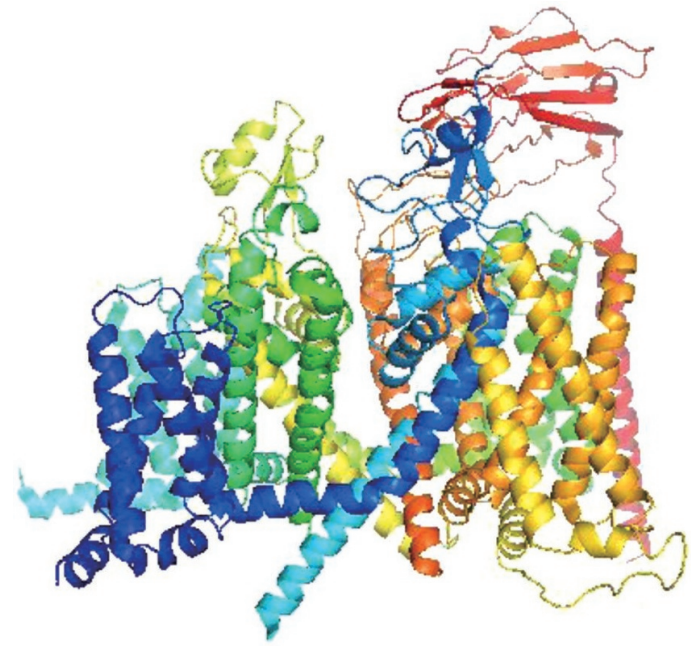

Fig. 5 Structure of the human Nav1.4- $\beta 1$ complex

angstroms ${ }^{[20]}$ (fig. 6). In the inactivated conformation, the fast-inactivation IlePhe-Met motif in III-IV linker inserts into the cavity between the S4-S5 restriction ring and the S6 helical bundle, providing support for the structural basis of "allosteric blocking" mechanism for fast inactivation. The binding mode for these two toxins by Nav1.7 is different from that for Dcla by NavPaS. Dcla projects into the extracellular cavity that is enclosed by the segments in VSD II and the PD. Indeed, the pore domain provides the support to anchor Dc1a, and then traps VSD II in a specific conformational state. In contrast, HWTX-IV and ProTx- II bind to the region that links S3 and S4 in VSD II. The distinction of binding mode provides the support to anchor the GMTs, and then locks the associated VSDs in functional states to modulate gating. Xu et al visualized ProTx2 in complex with VSD2 of Nav1.7 by X-ray crystallography and cryoelectron microscopy. ProTx2 was found to be trapped in activated and deactivated states of VSD2, which revealed a remarkable $\sim 10 \AA$

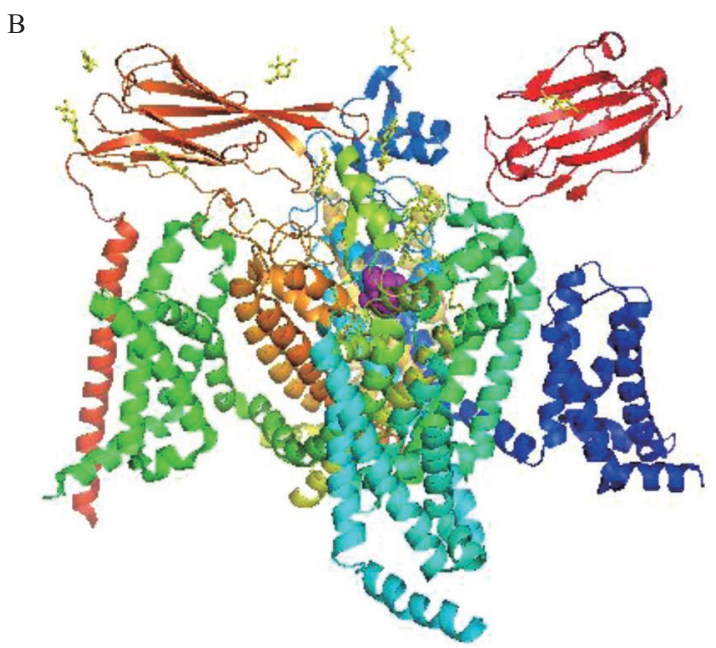

Fig. 6 Overall structures of human Nav1.7-HS and Nav1.7-PT are shown in A and B, respectively. 
translation of the S4 helix, and provided a structural framework for activation gating ${ }^{[34]}$.

Meanwhile, the published cryo-EM structure of human Nav1.2 bound to the m-conotoxin K IIIA (a peptidic pore blocker) in the presence of $\beta 2$ is at 3.0 angstroms $^{[21]}$ (fig. 7). The immunoglobulin domain in $\beta 2$ interacts with the shoulder of pore domain by a disulfide bond. KIIIA interacts with the specific residues in repeats I to III, revealing a molecular basis for K III A specificity. To date, Structural determination of the insect NavPaS in complex with Dc1a, the human Nav1.4- $\beta 1$ complex, the human Nav1.7 with two tarantula toxins, HWTX-IV and ProTx- II, and the human Nav1.2 with KIIIA in the presence of $\beta 2$, will provide a path toward structure-based drug discovery.

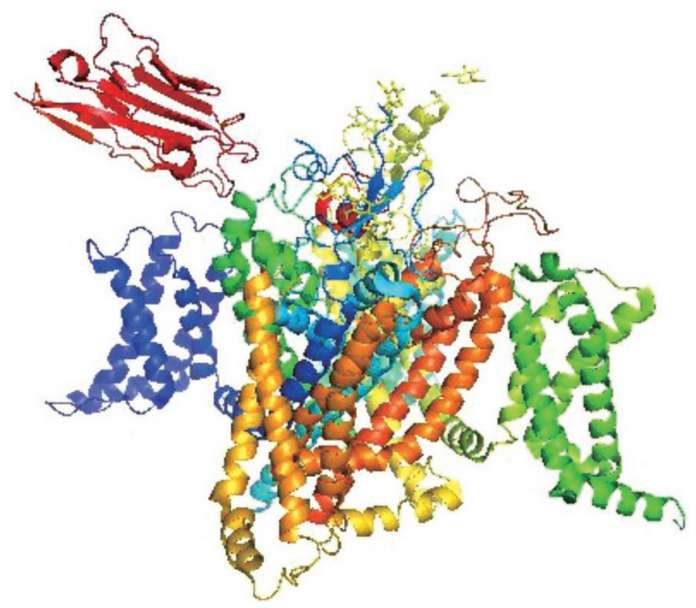

Fig. 7 Overall structure of the human Nav1.2- $\beta 2$ complex bound to K III A

\section{CHANNELOPATHIES RELATED TO NAV CHANNELS}

\subsection{Pain}

Pain, a physiological sensation, is perceived by organisms as it alerts organisms to dangers around the corner. Chronic neuropathic pain is associated with severe painful sensation which may derive from innocuous or acute pain stimuli, and it is often characterized by long duration and no particular trigger. Despite that there are numerous options for the treatment of chronic pain, it is still intractable to achieve the management of these syndromes. Discovery and development of new therapies for neuropathic pain represents a serious challenge. In fact, to date, it has been established that sodium channels are essential for the transition of pain sensation. In particular, at least three subtypes presented in human dorsal root ganglion (DRG) neurons, Nav1.7, Nav1.8 and Nav1.9 have been identified as the key players in transportation of pain signals ${ }^{[35-39]}$. Nav1.7 channels activate and inactivate rapidly. They, however, recover slowly from inactivation. Nav1.7 is considered a predominant Nav channel to produce substantial currents responding to depolarizations, which lead to neuronal firing produced by subthreshold stimuli.

Levels of activated p38 mitogen-activated protein kinase (MAPK) and signal-regulated kinase (ERK1/2) were increased in blind-ending axons of painful human neuromas with upregulated Nav1.7, suggesting that modulation of the Nav1.7 channel by MAPKs may be one of the mechanisms contributing to the development of ectopic discharges in nociceptors and neuroma associated pain in humans ${ }^{[40,41]}$. Genetic studies on patients with pain disorders revealed missense mutations in SCN9A encoding Nav1.7. Biophysical studies demonstrated that these mutations modulate Nav channel activation and/or inactivation properties and lead to hyperexcitability in DRG neurons ${ }^{[8]}$. Gainof-function mutations of NaV1.7 have been identified in patients with inherited erythromelalgia ${ }^{[42]}$, which is an autosomal dominant condition characterized by erythema of the extremities and burning pain, as well as in small fiber neuropathy known as painful neuropathy ${ }^{[43]}$, composed of small fiber related symptoms, including autonomic dysfunction, pain, loss of pinprick and allodynia, or hyperalgesia. However, loss-of-function mutations in Nav1.7 affected individuals unable to feel pain in the families with congenital insensitivity to pain (CIP).

Compared to Nav1.7 channels, Nav1.8 channels activate at more depolarized membrane potentials, and mediate the majority of the inward sodium currents during the depolarization of neuronal action potentials that are important for the transmission of action potentials as well as repetitive firing. Heterozygous gain-of-function mutations in SCN10A encoding Nav1.8 enable hyperexcitability and abnormal firing of DRG neurons, which is also associated with painful small-fiber neuropathies ${ }^{[44]}$. Mounting evidence indicates the nociceptive neuron function of Nav1.8. Similarly, mutations of Nav1.9 channels encoded by the gene SCN11A modulate Nav channels gating properties, and lead to depolarized potentials of membrane, likely resulting in abnormal excitability of DRG neurons ${ }^{[45]}$.

\subsection{Migraine}

Familial hemiplegic migraine type 3 (FHM3) is a severe autosomal dominant inherited subtype of FHM characterized by hemiparesis and visual aura during attacks ${ }^{[46]}$. Evidence from studies in patients has indicated that cortical spreading depression (CSD) is a torpidly propagating wave of neuronal and glial depolarization with an increase in potassium ion concentrations, stimulating trigeminovascular system from the meninges and activating downstream pain pathways, and possibly leading to headache. FHM3 is associated with dysfunction of Nav1.1, which varies from gain of function to complete loss of function ${ }^{[47]}$. The pathogenetic mechanisms have not been completely 
illuminated yet. A current hypothesis declares that abnormal voltage-gated sodium channels bring about neuronal hyperexcitability, increased release of neurotransmitter, and accumulated concentration of extracellular potassium, and ultimately lead to migraine.

\subsection{Epilepsy}

Epilepsy is a severe acquired or inherited disease characterized by abnormalities in neuronal activity, particularly, sustained firing of sodium-dependent action potentials due to a slow depolarized potential. Missense mutations in sodium channels encoding specific subunits including SCN1A (encoding the Nav1.1 $\alpha$-subunit), SCN2A (encoding the Nav1.2 $\alpha$-subunit), SCN9A (encoding the Nav1.7 $\alpha$-subunit), SCN1B (encoding $\beta 1$-subunit), and SCN3A (encoding the Nav1.3 $\alpha$-subunit $)^{[48]}$ are responsible for genetic epilepsy syndromes along with a wide range of severity, which include generalized epilepsy with febrile seizures plus (GEFS+), benign familial neonatal-infantile seizures, and Dravet syndrome. Missense mutations impair the excitability of inhibitory interneurons, leading to hyperexcitability and various epileptic seizures ${ }^{[49]}$. In particular, Nav1.1 channel is expressed in GABAergic interneurons synthesizing and releasing $\gamma$-aminobutyric acid (GABA, the major inhibitory neurotransmitter in the brain). Interneurons are important parts to regulate neuronal network excitability and synchronization of neuronal activity. Currently, the mechanism of epilepsy caused by the mutations is unclear, but it is thought to be related to the impaired generation of the current and action potential in GABAergic inhibitory neuronal $\mathrm{Na}^{+}$channels ${ }^{[50]}$.

\subsection{Cardiovascular Diseases}

The most common cardiac arrhythmogenic diseases associated with $\mathrm{Na}^{+}$channelopathies are long QT syndrome (LQT3) and Brugada syndromes $(\mathrm{BrSs})^{[51]}$. The significance of Nav channels in the normal cardiac electrical function has been underlined by the discovery of cardiovascular diseases linked with mutations in SCN5A, the gene encoding the Nav1.5 subunits $^{[52]}$. Most of these mutations destroy fast sodium channel inactivation, and impair the channel ability to close completely, thereby generating persistent sodium currents and prolonging the ventricular action potential ${ }^{[53]}$. In contrast, mutations that reduce INa decrease cardiac excitability, reduce velocity of electrical conduction, and in extreme cases, lead to BrSs, sick sinus syndrome, or combinations thereof. Genetic studies have also showed that SCN10A variants encoding Nav1.8 subunit could disturb Brugada syndrome and cardiac conduction, which demonstrated that the emergence of SCN10A may be act as a cardiac arrhythmia susceptibility gene ${ }^{[54]}$.

\subsection{Neurodegenerative Diseases}

Nav channels have been suggested to play a key role in processes involved in CNS disorders such as amyotrophic lateral sclerosis (ALS), the classical neurodegenerative disease Alzheimer's disease (AD), multiple sclerosis (MS), Parkinson's disease (PD), and Huntington disease (HD). Patients with MS show both negative (paresis, hypesthesia, and ataxia) and positive (pain and dysesthesia) symptoms. In addition, therapeutic approaches based on neuroprotection have been envisaged since decades to limit the neuronal damage consequent to severe insults, like traumatic brain injury (TBI) and intracerebral haemorrhage (ICH). Great efforts have being done to find methods effective on the progression of neurodegenerative diseases or secondary injuries. It was assumed that in those cases, featured by a reduced energy supply, leading to impaired action of $\mathrm{Na}^{+} / \mathrm{K}+$-ATP-depending pumps and depolarization of membranes, there may exist a greater persistent sodium ion conductance, most likely associated with Nav1.6. Sodium ions overload in the axon, accompanied with potassium ions efflux, may cause the accumulation of calcium ions via reversal of the $\mathrm{Na}+/ \mathrm{Ca}+$ exchanger, and trigger a succession of pathogenic cascade, which ends with neuronal loss. An persistent increase in $\mathrm{Na}+$ conductance, is probably to contribute to peripheral hyperexcitability in ALS, which leads to the classic symptoms of fasciculations and cramps, possibly resulting in the release of abnormal glutamate, which may play an important role in neurodegeneration ${ }^{[55]}$.

\subsection{Psychiatric Disorders}

Mutated Nav channels have been found in patients with certain psychiatric conditions, although these studies need to be confirmed by the analysis of a higher quantity of individuals. Data suggested that mutations in SCN8A may lead to cognitive deficits and SCN8A may be a potential susceptibility gene for Bipolar disorder ${ }^{[56]}$. In addition, one study on subjects with Dravet syndrome, a severe epilepsy often caused by mutations in SCN1A gene indicated that mutations in SCN1A gene can be responsible for both epilepsy and progressive severe mental impairment ${ }^{[57]}$.

\subsection{Cancer}

Analyses of various tumor tissues revealed that their intrinsic concentration of sodium ions is significantly higher than that of normal tissues and is closely related to the formation of tumors ${ }^{[58]}$. Nav channels, play an essential control function in cellular processes, including metastasis and angiogenesis, by regulating cell motility as well as the secretion of proteolytic enzymes. For example, the dysfunction of Nav1.5 channels and $\beta$ subunits can make cancer cells highly invasive. Over the past years, Nav channel inhibitors have displayed beneficial effects in diverse types of cancer, such as prostate cancer, lymphoma, mesothelioma and cervical cancer, and have been involved in regulation of carcinogenic process, 
including cell proliferation, invasiveness, and so on. However, the molecular mechanism involved in the regulation of Nav channels activity is still unclear. Currently, there are some hypotheses. The $\mathrm{Na}+$ influx activates the sodium-hydrogen antiporter (NHE1), leading to a low intracellular $\mathrm{pH}$, which enhances the activity of cathepsins, and therefore degrades the extracellular matrix ${ }^{[59,60]}$. After VGSC activation, mitochondria rapidly uptake $\mathrm{Na}^{+}$and release $\mathrm{Ca}^{2+}$ into cytosol $^{[61]}$, then promote the formation of invasive podosomes and enhance tumor cell invasion. The $\beta$ subunit changes the morphology and movement of tumor cells by adhesion mechanisms that are related to fyn kinase and $\mathrm{Na}^{+}$current ${ }^{[58]}$.

\section{ADVANCES IN THE DEVELOPMENT OF NAV CHANNEL BLOCKERS FOR THE TREATMENT OF CHANNELOPATHIES}

\subsection{As Pain-Relieving Drugs}

If the root cause of neuropathic pain is DRG hyperexcitability derived from enhanced $\mathrm{NaV}$ function, it stands to reason that selective $\mathrm{NaV}$ blockers should be anti-hyperalgesic. Experimental evidence indicated that local anesthetic (LA), class I cardiac antiarrhythmics, and antidepressants possess properties of releasing neuropathic pain by modulating Nav channels. Nevertheless, their actual efficacy to treat chronic pain is under investigation due to their off-target adverse effects such as cardiotoxicity, ataxia, confusion and sedation, which therefore narrow their therapeutic margin. Small molecules ICA-121431 and PF-04856264 (inhibiting Nav1.7 with an IC50 of $28 \mathrm{nmol} / \mathrm{L}$ ) bind to Nav voltage sensor of DIV ${ }^{[62]}$ and would certainly address the role of this novel binding site for pain control. Many companies are developing the second generation of molecules which are isoform-selective $\mathrm{NaV}$ blockers. For example, CNV1014802 (now in Phase III clinical trials for treatment of trigeminal neuralgia) ${ }^{[63]}$ from Convergence pharmaceuticals/Biogen Idec, and PF-05089771 from Pfizer are the most advanced clinical NaV1.7 blockers being tested in patients. Mutagenesis studies revealed that the compounds bind to the residues of S2 and S3 in the voltage-sensing domain IV, which has also been verified by the crystal structure of the Nav1.7 voltagesensing domain IV, an aryl sulfonamide complex ${ }^{[64]}$. Data for orally administered XEN402 targeting NaV1.7 have been reported in the peer-reviewed literature and the authors reported that XEN402 (i) significantly reduced the ability to induce pain from heat or exercise, as well as the amount of pain after induction $^{[65]}$, (ii) increased the time to reach maximal pain induction. Xenon developed XEN403 (a selective Nav1.7 blocker) as a backup candidate to XEN402, for the potential treatment of pain ${ }^{[66]}$. Phase I clinical trials on GDC-0276 and GDC-0310 from Genentech, Nav1.7-selective inhibitors for the treatment of pain ${ }^{[67]}$, have been completed in 2017. PF-04531083 from Pfizer is designed as NaV1.8 blockers $^{[68]}$, which is being tested into Phase II clinical trials. Moreover, several inhibitors including DSP-2230 and AZD-3161 have been advanced to clinical trials. In 2017, AM-0466 was identified as a Nav1.7-selective inhibitor, and showed a favorable pharmacokinetic profile in a licking time in a capsaicin-induced nociception model of pain ${ }^{[69]}$. In 2018, GX-201 and GX-585 was identified as Nav1.7selective inhibitors with the acylsulfonamide scaffold, and showed analgesic activity in inflammatory and neuropathic pain models ${ }^{[70]}$. Unfortunately, no selective blockers of NaV1.9 have been identified thus far. A feasible mechanism to regulate Nav channels in a selective pattern was exemplified by natural neurotoxins from the venom of snakes, scorpions and other animals ${ }^{[71,72]}$. These peptides have a variety of binding sites, particularly, the voltage sensors, which display less degree of observed sequence similarity than the pore-forming region. For example, D-conotoxins target DIV S3-S4 and slower inactivation, and scorpion $\alpha$-toxins could bind to DII-VSD ${ }^{[73]}$. In addition, $\mu$-SLPTX-Ssm6a, a peptide toxin from the venom of centipede, shows selectivity inhibition to Nav1.7 by transition from its activation to depolarized potentials. Notably, the modulation capability of HWTX-IV toxin is tailored on stabilizing the rest state of D II -VSD of Nav1.7, and blocking the channel ${ }^{[74]} \cdot \mu$-Conotoxin K III A, a peptide toxin from Cone snail, could bind to DI-DIV (pore region); $\mu$-Conotoxin MrVIA, a peptide toxin from Cone snail, could bind to D II and E2. The crystallographic study of the toxin-channel interaction could contribute to mechanistic insights into the modulation of Nav1.7 by blockers, and hold promise to make Nav1.7 as an irresistible target for drug design.

\subsection{Nav Channel Blockers for Migraine}

Antiepileptic drugs (AEDs), originally approved for the treatment of epilepsy, are one of the major categories of conventional drugs as sodium-channel blockers to treat migraine. Recent evidence from clinical trials suggests that some sodium channels blockers (for example, topiramate, lamotrigine and carbamazepine) seem to be efficacious for migraine therapy, reducing aura as well as migraine attacks. In particular, topiramate presents high efficacy in reducing the intensity, duration and frequency of migraine. Moreover, several studies showed both good tolerability and high efficacy for valproic acid ${ }^{[75]}$. Nevertheless, the detailed mechanism of action of these AEDs for the treatment of migraine need to be further explored.

\subsection{Nav Channel Blockers for Epilepsy}

Nav channels initiate action potentials in brain neurons, and sodium channel blockers are used in 
therapy of epilepsy. Anticonvulsants, such as phenytoin (PHT) and carbamazepine (CBZ), are broad-spectrum antiepileptic drugs for the treatment of seizures with a frequency-dependent and voltage-dependent manner, and have an exceptional range of anticonvulsant efficacy against both partial and generalized tonicclonic seizures. And they presumably act by attenuating sustained repetitive firing and voltage-gated sodium currents. Valproate and topiramate likely also act as sodium channel blockers as part of their therapeutic actions. Evidence has revealed that lacosamide represents an effective treatment for seizures by enhancing channel slow inactivation, and therefore, inhibiting Nav channels ${ }^{[76]}$. PHT and CBZ possess prominent ability of voltage-dependent blocking due to their preferential binding to the inactivated states of Nav channel. The narrow therapeutic range of these drugs have raised serious concerns for the treatment of epilepsy. Shaheen et al reported a potential sodium channel blocker, NSC403438, as a better candidate than PHT and CBZ ${ }^{[77]}$. It should be noted that sodium channel-blocking antiepileptic drugs are used broadly in the treatment of both generalized and partial seizures arising from different causes. But they are not effective in the treatment of absence seizures, driven by rhythmic firing in thalamic neurons that generate sleep rhythms.

\subsection{Nav Channel Blockers for Cardiovascular Diseases}

Class I antiarrhythmics, such as quinidine, procainamide, and disopyramide (class Ia agents) as well as mexiletine, lidocaine, phenytoin and tocainide (class Ib agents) and flecainide and propafenone (class Ic agents) play essential roles in the therapy of cardiac arrhythmias with other side effects due to their nonselective action ${ }^{[2]}$. Ranolazine, approved by the FDA in the USA, is useful for the therapy of chronic angina pectoris in the patients with ischemic heart disease. However, ranolazine is only weakly against the persistent sodium current and poorly selective versus several potassium channels. For these reasons, it is reasonable to suppose a combination of effects for this drug. GS-458967 and F15845 are the selective inhibitors of cardiac sodium current ${ }^{[53]}$. Moreover, F15845 plays a role in long- and shortterm cardioprotection after myocardial infarction. Meanwhile, F15845 does not affect peak sodium current in normal polarized myocytes at pharmacologically active doses. This feature distinguishes this compound from the commercially available NaV1.5 blockers (as class I antiarrhythmics).

\subsection{Nav Channel Blockers for Neurodegenerative Diseases}

Among compounds so far investigated, AEDs have displayed effective compete against cell apoptosis or necrosis in vitro and in vivo models of specific neurological diseases. Concerning the mechanism of activity, AED drugs counteract the abnormal neuronal excitability via modulating the action of ion channels, receptors and signaling pathways ${ }^{[78]}$. Among them, sodium channels are the main targets of AEDs in CNS. The increasing data have been reported that the damaged cognitive performance was related to reduced expression of Nav1.1 subtype in heterozygous SCN1A \pm mice. Meanwhile, reduced short-term memory was observed in otherwise healthy humans who carried SCN1A mutations, suggesting that a potential relevance between reduced expression of Nav1.1 and cognitive deficits. Therefore, Nav1.1 activators have potential for the symptomatic treatment of cognitive dysfunctions such as Alzheimer's disease and schizophrenia ${ }^{[79]}$. In addition, Nav1.2, Nav1.6, and Nav1.8 seem to be associated with pathophysiology of MS, probably as a result of dysregulated immune responses. Nav1.2 and Nav1.6 appear to play a key role in the restoration to neuronal conduction after axonal degeneration. The upregulation of Nav1.8 was observed in a mouse model of MS, in which cerebellar dysfunction also emerged. In addition, MS deficits in the mouse model could be reversed partially with A803467 (Nav1.8 blocker). Lidocaine and mexiletine, voltage-gated sodium channels blockers, have the ability to block the symptoms of MS, therefore acting selectively on fibers which mediate the positive symptoms. Although the mechanism of selective degeneration of motor neurons has not been entirely understood, several hypotheses were proposed that altered functionality of Nav channels may be the chief criminal. Evidence showed that riluzole, a drug to treat ALS, tended to block TTX sensitive Nav channels. Meanwhile, riluzole also shows a neuroprotective action in the rat model of Parkinson's disease. Remacemide and safinamide are also sodium channel blockers for the treatment of this disease in clinical trials. Experiment data have showed that the $\beta$-subunits of Nav channels are processed by BACE 1 and $\gamma$-secretase, which may provide new view for understanding of Alzheimer's disease.

\subsection{Nav Channel Blockers for Psychiatric Disorders}

Nav channel blockers have played a major role in the treatment of psychiatric disorders, for example, borderline personality disorder (BPD) and bipolar depression (BD). Compounds belong to the anticonvulsant class have displayed beneficial effects in therapeutic approaches to psychiatric disorders such as schizophrenia. Nevertheless, it is difficult to believe that blocking sodium channels expressed in the limbic system is the only mechanism of action of the drugs, and more studies for candidate mechanisms need to be carried out. Experimental data suggested that mutations in SCN8A may cause cognitive deficits ${ }^{[56]}$. Carbamazepine and lamotrigine are the only FDA approved drug molecules targeting voltage gated sodium channels for the treatment of psychiatric disorders. 
Carbamazepine was approved for the treatment of depression and acute mania in 2004. Lamotrigine has been approved for the treatment of BPD. Other sodium channel blockers like topiramate, oxcarbazepine, and zonisamide possess the capacity of stabilizing mood in schizoaffective and bipolar disorders. NW-3509, a new sodium channel blocker, showed efficacy in a broad spectrum of depression, psychosis, mania, and aggressiveness. After combination with antipsychotics, NW-3509 may improve their efficacy with a decrease of their dosage and side effects.

\subsection{Nav Channel Blockers for Cancer}

The main strategy for cancer therapy is to use sodium channel interfering drugs, especially acting on the NaV1.5 or NaV1.7 channel ${ }^{[80]}$. Sodium channel inhibitors proven to suppress tumor progression are the neurotoxin TTX, which inhibits cell invasion in breast in vitro. Phenytoin and a-hydroxy-aphenylamides were proven useful to treat prostate cancer. In addition, riluzole, ranolazine, phenytoin, lidocaine, and carbamazepine have shown cell metastatic blocking behavior in in-vitro models. However, it is unrealistic to use these existing sodium channel blocker to treat cancer, because many of them, for example, anticonvulsants or antidepressants, could travel across the blood-brain barrier and function on isoforms without selectivity, thus causing many side effects.

\section{CONCLUSION}

In short, mutations in genes encoding for different Nav channels have been approved to play a key role in pathological disorders. Moreover, phylogenetic analyses have ensured the relationship between these channels with Nav channels from an evolutionary perspective. However, Nav channel blockers have typically showed poor selectivity to specific isoforms due to a high degree of sequence similarity between the subtypes, thus raising potential risks of many side effects. Nevertheless, Nav channels endowed with multifarious therapeutic potential could serve as interesting targets for drug discovery in the emerging therapeutic fields. To date, structures of the insect NavPaS in complex with Dc1a, the human Nav1.4- $\beta 1$ complex, the human Nav1.7 with two tarantula toxins, HWTX-IV and ProTx- II , and the human Nav1.2 with $\mathrm{K}$ III A in the presence of $\beta 2$ have been reported. These structures help understand their pharmacological profiles, as well as give insight into the molecular basis of sodium functional mechanism for ion permeation and provide a path toward structure-based drug discovery. In spite of these remarkable studies holding promise to structure-based drug design, it still represents great challenges due to the transitions between working states of voltage-dependent sodium channels entailing intricate conformational changes and reconfiguration of interactions between structural elements by direct or allosteric effects. Based on this, great efforts need to be conducted to interpret the interior relations of Nav mutations and corresponding structures based on the published specific structures for the treatment of channelopathies.

\section{Open Access}

This article is licensed under a Creative Commons Attribution 4.0 International License https://creativecommons.org/licenses/by/4.0/), which permits use, sharing, adaptation, distribution and reproduction in any medium or format, as long as you give appropriate credit to the original author(s) and the source, provide a link to the Creative Commons licence, and indicate if changes were made. The images or other third party material in this article are included in the article's Creative Commons licence, unless indicated otherwise in a credit line to the material. If material is not included in the article's Creative Commons licence and your intended use is not permitted by statutory regulation or exceeds the permitted use, you will need to obtain permission directly from the copyright holder. To view a copy of this licence, visit http://creativecommons.org/licenses/by/4.0/.

\section{Conflict of Interest Statement}

The authors claim that the researchers in this study have no conflict of interest.

\section{REFERENCES}

1 Ahern CA, Payandeh J, Bosmans F, et al. The hitchhiker's guide to the voltage-gated sodium channel galaxy. J Gen Physiol, 2016,147(1):1-24

2 Carnevale V, Klein ML. Small molecule modulation of voltage gated sodium channels. Curr Opin Struct Biol, 2017,43:156-162

3 Huang W, Liu M, Yan SF, et al. Structure-based assessment of disease-related mutations in human voltage-gated sodium channels. Protein Cell, 2017,8(6):401-438

4 Catterall WA. Forty Years of Sodium Channels: Structure, Function, Pharmacology, and Epilepsy. Neurochem Res, 2017,42(9):2495-2504

5 Catterall WA. Sodium channels, inherited epilepsy, and antiepileptic drugs. Annu Rev Pharmacol Toxicol, 2014,54:317-338

6 Rubinstein M, Patowary A, Stanaway IB, et al. Association of rare missense variants in the second intracellular loop of NaV1.7 sodium channels with familial autism. Mol Psychiatry, 2018,23(2):231-239

7 Luiz AP, Wood JN. Sodium Channels in Pain and Cancer: New Therapeutic Opportunities. Adv Pharmacol, 2016,75:153-178

8 Dib-Hajj SD, Yang Y, Black JA, et al. The $\mathrm{Na}(\mathrm{V}) 1.7$ sodium channel: from molecule to man. Nat Rev Neurosci, 2013,14(1):49-62

9 Dib-Hajj SD, Cummins TR, Black JA, et al. Sodium channels in normal and pathological pain. Annu Rev Neurosci, 2010,33:325-347

10 Pedraza Escalona M, Possani LD. Scorpion beta-toxins and voltage-gated sodium channels: interactions and effects. Front Biosci (Landmark Ed), 2013,18:572-587 
11 Bagal SK, Marron BE, Owen RM, et al. Voltage gated sodium channels as drug discovery targets. Channels (Austin), 2015,9(6):360-366

12 Payandeh J, Scheuer T, Zheng N, et al. The crystal structure of a voltage-gated sodium channel. Nature, 2011,475(7356):353-358

13 Payandeh J, Gamal El-Din TM, Scheuer T, et al. Crystal structure of a voltage-gated sodium channel in two potentially inactivated states. Nature, 2012,486(7401):135-139

14 Zhang X, Ren W, DeCaen P, et al. Crystal structure of an orthologue of the $\mathrm{NaChBac}$ voltage-gated sodium channel. Nature, 2012,486(7401):130-134

15 McCusker EC, Bagneris C, Naylor CE, et al. Structure of a bacterial voltage-gated sodium channel pore reveals mechanisms of opening and closing. Nat Commun, 2012,3:1102-1109

16 Shen H, Zhou Q, Pan X, et al. Structure of a eukaryotic voltage-gated sodium channel at near-atomic resolution. Science, 2017,355(6328):eaal4326

17 Yan Z, Zhou Q, Wang L, et al. Structure of the Nav1.4beta1 Complex from Electric Eel. Cell, 2017,170(3):470482 e11

18 Shen H, Li Z, Jiang Y, et al. Structural basis for the modulation of voltage-gated sodium channels by animal toxins. Science, 2018,362(6412): eaau2596

19 Pan X, Li Z, Zhou Q, et al. Structure of the human voltage-gated sodium channel Nav1.4 in complex with beta1. Science, 2018,362(6412):eaau2486

20 Shen H, Liu D, Wu K, et al. Structures of human Nav1.7 channel in complex with auxiliary subunits and animal toxins. Science, 2019,363(6433):1303-1308

21 Pan X, Li Z, Huang X, et al. Molecular basis for pore blockade of human $\mathrm{Na}(+)$ channel Nav1.2 by the muconotoxin KIIIA. Science, 2019,363(6433):1309-1313

22 Catterall WA. Voltage-gated sodium channels at 60: structure, function and pathophysiology. J Physiol, 2012,590(11):2577-2589

23 Corry B, Thomas M. Mechanism of ion permeation and selectivity in a voltage gated sodium channel. $\mathrm{J}$ Am Chem Soc, 2012,134(3):1840-1846

24 de Lera Ruiz, M, Kraus RL. Voltage-Gated Sodium Channels: Structure, Function, Pharmacology, and Clinical Indications. J Med Chem, 2015,58(18):70937118

25 Bagal SK, Brown AD, Cox PJ, et al. Ion channels as therapeutic targets: a drug discovery perspective. J Med Chem, 2013,56(3):593-624

26 Stock L, Souza C, Treptow W. Structural basis for activation of voltage-gated cation channels. Biochemistry, 2013,52 (9):1501-1513

27 Stock L, Delemotte L, Carnevale V, et al. Conduction in a biological sodium selective channel. J Phys Chem B, 2013,117(14):3782-3789

28 Favre I, Moczydlowski E, Schild L. On the structural basis for ionic selectivity among $\mathrm{Na}^{+}, \mathrm{K}^{+}$, and $\mathrm{Ca}^{2+}$ in the voltage-gated sodium channel. Biophys J, 1996,71(6): 3110-3125

29 Shaya D, Findeisen F, Abderemane-Ali F, et al. Structure of a prokaryotic sodium channel pore reveals essential gating elements and an outer ion binding site common to eukaryotic channels. J Mol Biol, 2014,426(2):467-
483

30 Palovcak E, Delemotte L, Klein ML, et al. Evolutionary imprint of activation: the design principles of VSDs. J Gen Physiol, 2014,143(2):145-156

31 Patton DE, West JW, Catterall WA, et al. Amino acid residues required for fast $\mathrm{Na}(+)$-channel inactivation: charge neutralizations and deletions in the III-IV linker. Proc Natl Acad Sci USA, 1992,89(22):10905-10909

32 Kalia J, Milescu M, Salvatierra J, et al. From foe to friend: using animal toxins to investigate ion channel function. J Mol Biol, 2015,427(1):158-175

33 Laedermann CJ, Syam N, Pertin M, et al. beta1- and beta3- voltage-gated sodium channel subunits modulate cell surface expression and glycosylation of Nav1.7 in HEK293 cells. Front Cell Neurosci, 2013,7:137-158

$34 \mathrm{Xu} \mathrm{H}, \mathrm{Li}$ T, Rohou A, et al. Structural Basis of Nav1.7 Inhibition by a Gating-Modifier Spider Toxin. Cell, 2019,176(4):702-715

35 Hoeijmakers JG, Faber CG, Merkies IS, et al. Painful peripheral neuropathy and sodium channel mutations. Neurosci Lett, 2015,596:51-59

36 Habib AM, Wood JN, Cox JJ. Sodium channels and pain. Handb Exp Pharmacol, 2015, 227:39-56

37 Dib-Hajj SD, Black JA, Waxman SG. NaV1.9: a sodium channel linked to human pain. Nat Rev Neurosci, 2015,16(9):511-519

38 Sun S, Jia Q, Zenova AY, et al. Identification of Selective Acyl Sulfonamide-Cycloalkylether Inhibitors of the Voltage-Gated Sodium Channel (NaV) 1.7 with Potent Analgesic Activity. J Med Chem, 2019,62(2):908-927

39 Lee JH, Park CK, Chen G, et al. A monoclonal antibody that targets a NaV1.7 channel voltage sensor for pain and itch relief. Cell, 2014,157(6):1393-1404

40 Black JA, Nikolajsen L, Kroner K, et al. Multiple sodium channel isoforms and mitogen-activated protein kinases are present in painful human neuromas. Ann Neurol, 2008,64(6):644-653

41 Hameed S. Nav1.7 and Nav1.8: Role in the pathophysiology of pain. Mol Pain, 2019,15:1-11

42 Cregg R, Laguda B, Werdehausen R, et al. Novel mutations mapping to the fourth sodium channel domain of Nav1.7 result in variable clinical manifestations of primary erythromelalgia. Neuromolecular Med, 2013,15(2):265-278

43 Faber CG, Hoeijmakers JG, Ahn HS, et al. Gain of function Nanu1.7 mutations in idiopathic small fiber neuropathy. Ann Neurol, 2012,71(1):26-39

44 Faber CG, Lauria G, Merkies IS, et al. Gain-of-function Nav1.8 mutations in painful neuropathy. Proc Natl Acad Sci USA, 2012,109(47):19444-19449

45 Zhang XY, Wen J, Yang W, et al. Gain-of-function mutations in SCN11A cause familial episodic pain. Am J Hum Genet, 2013,93(5):957-966

46 Shao N, Zhang H, Wang X, et al. Familial Hemiplegic Migraine Type 3 (FHM3) with an SCN1A Mutation in a Chinese Family: A Case Report. Front Neurol, 2018,9:976-984

47 Cestele S, Labate A, Rusconi R, et al. Divergent effects of the T1174S SCN1A mutation associated with seizures and hemiplegic migraine. Epilepsia, 2013,54(5):927935

48 Mantegazza M, Curia G, Biagini G, et al. Voltage- 
gated sodium channels as therapeutic targets in epilepsy and other neurological disorders. Lancet Neurol, 2010,9(4):413-424

49 Fujiwara T, Sugawara T, Mazaki-Miyazaki E, et al. Mutations of sodium channel alpha subunit type 1 (SCN1A) in intractable childhood epilepsies with frequent generalized tonic-clonic seizures. Brain, 2003,126(Pt 3):531-546

50 Catterall WA, Kalume F, Oakley JC. NaV1.1 channels and epilepsy. J Physiol, 2010,588(Pt 11):1849-1859

51 Tao YX. Pharmacology and therapeutics of constitutively active receptors. Preface. Adv Pharmacol, 2014,70:ix-x

52 Han D, Tan H, Sun C, et al. Dysfunctional Nav1.5 channels due to SCN5A mutations. Exp Biol Med (Maywood), 2018,243(10):852-863

53 Antzelevitch C, Nesterenko V, Shryock JC, et al. The role of late I $\mathrm{Na}$ in development of cardiac arrhythmias. Handb Exp Pharmacol, 2014,221:137-168

54 van den Boogaard M, Smemo S, Burnicka-Turek $\mathrm{O}$, et al. A common genetic variant within SCN10A modulates cardiac SCN5A expression. J Clin Invest, 2014,124(4):1844-1852

55 Vucic S, Kiernan MC. Upregulation of persistent sodium conductances in familial ALS. J Neurol Neurosurg Psychiatry, 2010,81(2):222-227

56 Wang Y, Zhang J, Liu B, et al. Genetic polymorphisms in the SCN8A gene are associated with suicidal behavior in psychiatric disorders in the Chinese population. World J Biol Psychiatry, 2010,11(8):956-963

57 Riva D, Vago C, Pantaleoni C, et al. Progressive neurocognitive decline in two children with Dravet syndrome, de novo SCN1A truncations and different epileptic phenotypes. Am J Med Genet A, 2009,149A(10):2339-2345

58 Mao W, Zhang J, Korner H, et al. The Emerging Role of Voltage-Gated Sodium Channels in Tumor Biology. Front Oncol, 2019,9:124-143

59 Busco G, Cardone RA, Greco MR, et al. NHE1 promotes invadopodial ECM proteolysis through acidification of the peri-invadopodial space. FASEB J, 2010,24(10):3903-3915

60 Brisson L, Gillet L, Calaghan S, et al. $\mathrm{Na}(\mathrm{V}) 1.5$ enhances breast cancer cell invasiveness by increasing NHE1-dependent $\mathrm{H}(+)$ efflux in caveolae. Oncogene, 2011,30(17):2070-2076

61 Yang M, Kozminski DJ, Wold LA, et al. Therapeutic potential for phenytoin: targeting $\mathrm{Na}(\mathrm{v}) 1.5$ sodium channels to reduce migration and invasion in metastatic breast cancer. Breast Cancer Res Treat, 2012,134(2):603615

62 McCormack K, Santos S, Chapman ML, et al. Voltage sensor interaction site for selective small molecule inhibitors of voltage-gated sodium channels. Proc Natl Acad Sci U S A, 2013,110(29):E2724-E2732

63 Deuis JR, Wingerd JS, Winter Z, et al. Analgesic Effects of GpTx-1, PF-04856264 and CNV1014802 in a Mouse Model of NaV1.7-Mediated Pain. Toxins (Basel), 2016, 8(3):78-104

64 Ahuja S, Mukund S, Deng L, et al. Structural basis of Nav1.7 inhibition by an isoform-selective smallmolecule antagonist. Science, 2015,350(6267):aac5464
65 Goldberg YP, Price N, Namdari R, et al. Treatment of $\mathrm{Na}(\mathrm{v}) 1.7$-mediated pain in inherited erythromelalgia using a novel sodium channel blocker. Pain, 2012,153(1):80-85

66 Bagal SK, Chapman ML, Marron BE, et al. Recent progress in sodium channel modulators for pain. Bioorg Med Chem Lett, 2014,24(16):3690-3699

67 Emery EC, Luiz AP, Wood JN. Nav1.7 and other voltage-gated sodium channels as drug targets for pain relief. Expert Opin Ther Targets, 2016,20(8):975-983

68 Bagal SK, Bungay PJ, Denton SM, et al. Discovery and Optimization of Selective Nav1.8 Modulator Series That Demonstrate Efficacy in Preclinical Models of Pain. ACS Med Chem Lett, 2015,6(6):650-654

69 Graceffa RF, Boezio AA, Able J, et al. Sulfonamides as Selective NaV1.7 Inhibitors: Optimizing Potency, Pharmacokinetics, and Metabolic Properties to Obtain Atropisomeric Quinolinone (AM-0466) that Affords Robust in Vivo Activity. J Med Chem, 2017,60(14):59906017

70 Bankar G, Goodchild SJ, Howard S, et al. Selective NaV1.7 Antagonists with Long Residence Time Show Improved Efficacy against Inflammatory and Neuropathic Pain. Cell Rep, 2018,24(12):3133-3145

71 Catterall WA. Ion channel voltage sensors: structure, function, and pathophysiology. Neuron, 2010,67(6):915928

72 Cestele S, Qu Y, Rogers JC, et al. Voltage sensortrapping: enhanced activation of sodium channels by beta-scorpion toxin bound to the S3-S4 loop in domain II. Neuron, 1998,21(4):919-931

73 Heinemann SH, Leipold E. Conotoxins of the O-superfamily affecting voltage-gated sodium channels. Cell Mol Life Sci, 2007,64(11):1329-1340

74 Xiao Y, Blumenthal K, Jackson JO, 2nd, et al. The tarantula toxins ProTx-II and huwentoxin-IV differentially interact with human Nav1.7 voltage sensors to inhibit channel activation and inactivation. Mol Pharmacol, 2010,78(6):1124-1134

75 Chiossi L, Negro A, Capi M, et al. Sodium channel antagonists for the treatment of migraine. Expert Opin Pharmacother, 2014,15(12):1697-1706

76 Errington AC, Stohr T, Heers C, et al. The investigational anticonvulsant lacosamide selectively enhances slow inactivation of voltage-gated sodium channels. Mol Pharmacol, 2008,73(1):157-169

77 Shaheen U, Akka J, Hinore JS, et al. Computer aided identification of sodium channel blockers in the clinical treatment of epilepsy using molecular docking tools. Bioinformation, 2015,11(3):131-137

78 Caccamo D, Pisani LR, Mazzocchetti $\mathrm{P}$, et al. Neuroprotection as a Potential Therapeutic Perspective in Neurodegenerative Diseases: Focus on Antiepileptic Drugs. Neurochem Res, 2016,41(1-2):340-352

79 Jensen HS, Grunnet M, Bastlund JF. Therapeutic potential of $\mathrm{Na}(\mathrm{V}) 1.1$ activators. Trends Pharmacol Sci, 2014,35(3):113-118

80 Black JA, Waxman SG. Noncanonical roles of voltagegated sodium channels. Neuron, 2013,80(2):280-291

(Received Dec. 12, 2018; revised Sep. 2, 2019) 